depressive disorder might be associated with free-radical damage and subsequent lipid system abnormalities.

Maes et al (1996) found a significant decrease in the $\omega 3$ fractions in cholesteryl esters in patients with major depression compared with minor depression or healthy controls. They also reported a significantly increased ratio of arachidonic acid to eicosapentaenoic acid in both cholesteryl esters and phospholipids. Peet et al (1998) found depleted total $\omega 3$ PUFA and docosahexaenoic acid in the red blood cell membrane of patients with depression. They have argued that the changes in serotonin receptor number and function caused by changes in PUFA provide the theoretical rationale connecting fatty acids with current neurotransmitter and receptor theories of depression. The possibility of dietary supplementation of fatty acids has not received enough attention in clinical trials of depression, although there are anecdotal reports of their efficacy in stress-induced aggressive behaviour (Hamazaki et al, 1996).

Hence, advances in lipid neurochemistry may prove useful not only in understanding schizophrenia, but also in understanding and potentially treating other psychiatric diseases including depressive disorders.

Finkel, M. S., Thode, F. L., Pollock, B. J., et al (1996) Paroxetine: a novel nitric oxide synthetase inhibitor. Psychopharmocology Bulletin, 32, 653-658.

Hamazald, T, Samarald, S., ltomura, M., et of (1996) The effect of docosahexaenoic acid on aggression in young adults, Journal of Clinical Investigation. 97. $1129-1134$.

Maes, M., Smith, R., Christophe, A., et al (1996) Fatty acid composition in major depression: Decreased $\omega 3$ fractions in cholesteryl esters and increased C 20:

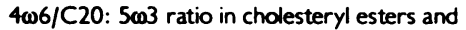
phospholipids. Journal of Affective Disorders, 38, 35-46.

Peet, M., Murphy, B., Shay, J., ot al (1990) Depletion of omega -3 fatty acid levels in red blood cell membrane of depressive patients. Biological Psychiatry, 43, 315-319.

Smith, R. S. (1991) The macrophage theory of depression. Medical Hypothesis, 35, 298-306.

Walleer, N. P., Fox, H. C. \& Whalley, L. J. (1999) Lipids and schizophrenia. British fournal of Psychiatry, 174. 10I-104.

S. Swain Department of Neurochemistry, Institute of Human Behaviour \& Allied Sciences (IHBAS), Shahdara, Delhi II0095, India

C. Pradhan Department of Psychiatry, Institute of Human Behaviour \& Allied Sciences (IHBAS), Shahdara, Delhi II0095, India

\section{Family functioning and adolescent anorexia nervosa}

Sir: We read with interest the article on difficulties in family functioning and adolescent anorexia nervosa by Gowers \& North (1999). This is an important area of research and we applaud the authors for a data-based publication in the Journal. We would, however, like to offer an alternative interpretation of their findings.

There were no significant associations between global ratings of family functioning by clinician, parent and patient and severity of anorexia nervosa; however, the authors conclude that there are some associations with separate sub-scales of family functioning. It is important first to highlight that these correlations are very small. The patients' opinion that family difficulties, such as resolving problems, increase with their increasing weight or sexual activity, is not an unexpected finding. Most patients with anorexia experience a re-emergence of feelings, particularly those of a conflictual nature, as their weight increases. This is particularly evident around sexual feelings, with which a significant proportion of adolescents with anorexia struggle. The experience of a decrease in difficult feelings with weight loss reinforces the usefulness of anorexia as a defence. Their heightened awareness of family difficulties with weight increase and clinical recovery is then to be expected.

The authors report no correlation between change in family functioning and clinical change as defined by the MorganRussell scale (1988). They have not given the range of change scores on their measure of family functioning, or the standard deviation. These would have facilitated the reader's assessment of the findings. The authors previously reported family functioning as predicting outcome of the anorexic illness at one year. That observation and their current finding of minimal change in family functioning during treatment cannot be offered as evidence for or against family dynamics as contributory factors in the aetiology of anorexia. We suggest that it would be more beneficial to contrast engagement or ability to effectively use therapy with family functioning. Anorexia is not caused by disturbed family dynamics; severity and aetiology of the illness is multi-factorial. However, the capacity of a family to use a treatment may be severely diminished by their own internal and difficult dynamics. The Family
Assessment Device may merely be a measure of engagement which, in turn, will impact on outcome of an anorexic illness.

Gowers, S. \& North, C. (1999) Difficulties in family functioning and adolescent anorexia nervosa. British Journal of Psychiatry, 174, 63-66.

Morzan, H. G. \& Hayward, A. E. (1988) Clinical assessment of anorexia nervosa. The Morgan-Russell outcome assessment schedule. British journal of Psychiatry, 152, 367-37I.

C. Dare Institute of Psychiatry, De Crespigny Park, Denmark Hill, London SE5 8AF

A. Key St George's Hospital Medical School, Cranmer Terrace, Tooting, London SWI7 ORE

Authors' reply: We are grateful to Drs Dare and Key for their interest in and comments on our paper. They are right to draw attention to the limitations of our findings and to put the role of family difficulties in perspective alongside other variables in the multi-factorial aetiology of anorexia nervosa.

However, we wish to take issue with two of their points. First, although we agree with Dare \& Key that anorexia nervosa can be interpreted as a defensive strategy against difficult feelings, this view is not universally held and, where it is, it is usually construed as an individual defence, rather than serving to reduce difficulties within the family. We contend that this is a new finding.

Second, and more importantly, the assertion that family dynamics do not cause anorexia nervosa is surely unhelpful. Although in our series, our clinical experience led us to believe that the families were without exception caring, conscientious and determined to be good families, surely family life has to be considered one of the major influences on adolescent development. If family factors can contribute to positive aspects of development like resilience, confidence, educational success and the ability to form satisfactory interpersonal relationships, then surely they can play a part in the development of negative ones. We suggest that the following features, impacting on family functioning, may, among others, be very likely to contribute to the development of eating disorders: parental disharmony and separation; parental eating disorders; death and severe illness in firstdegree relatives; emotional, physical and sexual abuse; and delinquency and unwanted pregnancies in siblings and their perceived affects on family life. 